\title{
FutureJournal
}

\section{Teoria dos Jogos: Identificação do Ponto de Equilíbrio de Nash em Jogos Bimatriciais em Indicadores Econômicos e Sociais}

\author{
Fernanda Kreuzberg \\ Mestre em Ciências Contábeis pela Universidade Regional de Blumenau - FURB, Brasil \\ fernandakreuzberg@gmail.com \\ Nelson Hein \\ Doutor em Engenharia de Produção pela Universidade Federal de Santa Catarina (UFSC), \\ Brasil \\ hein@furb.br
}

\section{Moacir Manoel Rodrigues Junior}

Doutor em Métodos Numéricos em Engenharia pela Universidade Federal do Paraná, Brasil martinho@usp.br

\section{RESUMO}

Atualmente as empresas precisam pensar dentro de seu sistema de gestão em satisfazer não apenas seus interesses econômicos, mas também seus interesses sociais visando à sustentabilidade e à perenidade das atividades. Nesta pesquisa, o objetivo é identificar o ponto de equilíbrio de Nash no jogo bimatricial de indicadores econômicos e sociais das empresas do setor de Utilidade Pública listadas na BM\&FBovespa. Realizou-se a pesquisa com uma amostra de 26 empresas. Para avaliar o desempenho econômico utilizaram-se os indicadores de rentabilidade e mercado; para a avaliação de desempenho social, utilizaram-se indicadores oriundos da DVA (Demonstração do Valor Adicionado). Na estimação dos ganhos entre os indicadores, utilizaram-se os parâmetros padronizados da regressão linear múltipla. Nos resultados obtidos, destacou-se a existência de pelo menos um ponto de equilíbrio para os anos analisados, e em especial nos modelos propuseram-se dois pontos de equilíbrio para 2009 e 2011, e três para 2010. Destaca-se que, no modelo proposto, confirmou-se a hipótese da existência de pelo menos um ponto de equilíbrio. Desse modo, conseguiu-se satisfazer o objetivo da pesquisa de que é possível atender tanto aos aspectos econômicos quanto aos aspectos sociais das empresas quando se tomam como informações básicas os indicadores. 


\section{FutureJournal}

PALAVRAS-ChAVE: Teoria dos jogos. Ponto de equilíbrio de Nash. Jogos Bimatriciais. Desempenho econômico. Desempenho Social.

\section{Game Theory: The Identification of the Nash Equilibrium in Bi- Matrix Games in Economic and Social Indicators}

\section{ABSTRACT}

Today, companies need to think within your management system to meet not only the economic interests of the same, but satisfy the social interests of the company glimpsing the sustainability and longevity of the activities. The objective of the research is to identify the Nash equilibrium in the game bi-matrix of economic and social indicators of companies in the public utilities sector listed on the BM\&FBovespa. For evaluate of the economic performance we used the profitability and market indicators, and for evaluate social performance, we used the indicators derived from the DVA. In the estimation of earnings among the indicators, we used the standardized parameters of multiple linear regression. The results highlighted the existence of at least one equilibrium point for the years analyzed, and in particular the models proposed two equilibrium points for 2009 and 2011, and three points for 2010. It is noteworthy that the proposed model confirmed the hypothesis of the existence of at least one equilibrium point, and so the search can meet your goal that you can meet both the economic aspects and social aspects of business when it takes as the basic information indicators.

KEY-WORDS: Game theory. Nash equilibrium. Games bi-matrix. Economic performance. Social performance. 


\section{INTRODUÇÃO}

Durante a última década, houve um aumento na pressão exercida sobre as empresas para que ampliem seu foco na sustentabilidade e na responsabilidade social dos negócios, para além do desempenho financeiro (Lee \& Saen, 2012). O conceito de desenvolvimento sustentável tornou-se popular na década de 1980 , devido à definição apresentada em 1987, pela World Commission on Environment and Development (WCED, 1987) das Nações Unidas, documento também conhecido como Relatório de Brundtland. A definição apresentada envolve um conceito ético em relação ao combate à pobreza e à proteção do meio ambiente, compreendendo o nível macro (Baumgartner \& Ebner, 2010; Clarke-Sather, Hutchins, Zhang, Gershenson \& Sutherland, 2011).

Conforme Claro, Claro e Amâncio (2008), após a definição da Comissão Brundtland, surgiram diversas outras, e muitas outras existirão no futuro; porém, o ponto em comum está na análise das dimensões que compõem a sustentabilidade.

Ebner e Baumgartner (2006) afirmam que no decorrer das últimas décadas a sustentabilidade tornou-se um termo muito popular na economia. Lee e Saen (2012) confirmam que a adoção de práticas de sustentabilidade garante uma vantagem competitiva bem como a promulgação da eficiência das empresas em um cenário turbulento de negócios. Da mesma forma, Ioannou e Serafeim (2012) salientam que a adoção de práticas sustentáveis pelas empresas, além de ocasionar uma vantagem competitiva e incrementar o desempenho financeiro de longo prazo, pode Ihes propiciar uma melhora no valor econômico gerado.

A ciência vem reforçando o foco da sustentabilidade empresarial, principalmente no que tange à diferenciação dos termos de sustentabilidade (especificamente a dimensão social) e responsabilidade social. Ebner e Baumgartner (2006) efetuaram um levantamento bibliográfico e apontaram como sendo comum a discussão dessa diferenciação dos termos, e que, na visão de diversos autores, ambos os conceitos apresentam abordagens distintas. Nesse sentido, Baumgartner e Ebner (2010) corroboram que a 
abordagem do desenvolvimento sustentável incorporado por uma organização é denominado de sustentabilidade empresarial, possuindo três pilares: econômico, ambiental e social.

A sustentabilidade corporativa é definida a partir do atendimento das necessidades atuais, diretas e indiretas dos stakeholders, bem como do atendimento das necessidades dos futuros stakeholders da empresa (Dyllick \& Hockerts, 2002). Portanto, para atender a esse objetivo, as empresas necessitam manter seu crescimento econômico, social e ambiental. Nesse sentido, surge o conceito do triple-bottom line, em que prevalece a perspectiva de integração dos aspectos econômicos, sociais e ambientais.

De acordo com Krajnc e Glavic (2005), para um acompanhamento dos resultados de suas atividades, as empresas utilizavam apenas indicadores financeiros. Porém, para atender às exigências de seus agentes (fornecedores, empregados, bancos, seguradoras, acionistas, sociedade em geral), as empresas começaram a adotar práticas mais sustentáveis. Conforme Callado (2010) e Callado e Fensterseifer (2010), a motivação para a adoção de práticas sustentáveis consiste na continuidade das empresas que não é mais garantida apenas pela lucratividade. Influenciada por ações de sustentabilidade, a empresa pode estender suas atividades por um período maior, gerando um aumento no valor de mercado das ações.

Em seu estudo, Lo (2010) buscou analisar a eficiência das empresas norte-americanas sustentáveis em comparação com as empresas não sustentáveis, pela avaliação da rentabilidade, utilizando o modelo de Análise Envoltória de Dados (DEA). A autora concluiu que as empresas sustentáveis apresentaram um melhor desempenho nos modelos de rentabilidade. 0 estudo compreende sustentabilidade ambiental a existência deste equilíbrio entre as ações de cunho econômico para com as ações de cunho social e ambiental.

Diante das diversas abordagens direcionadas para o conceito de sustentabilidade empresarial, o presente estudo adota o tradicional tripé da sustentabilidade, composto pelas dimensões econômica, social e ambiental. A adoção desse tripé justifica-se por ser essa a classificação mais consolidada na literatura até o momento. Efetuou-se a exclusão da dimensão ambiental no contexto da sustentabilidade empresarial, pelo fato de a sustentabilidade 
econômica ser o primeiro critério de análise (devido a seu foco pautado na maximização dos resultados da empresa). Além disso, a dimensão social representa o mais alto critério de responsabilidade social, de maneira a contribuir com o bem-estar da sociedade, incluindo o conjunto de stakehoders (Aupperle, Carroll \& Hatfield, 1985; Carroll, 1991).

Diante do exposto, apresenta-se a seguinte questão: Qual o ponto de equilíbrio de Nash, no jogo bimatricial de indicadores econômicos e indicadores sociais das empresas do setor de utilidade pública listadas na BM\&FBovespa? No intuito de responder essa problemática, na presente pesquisa, o objetivo é identificar o ponto de equilíbrio de Nash no jogo bimatricial de indicadores econômicos e indicadores sociais das empresas do setor de utilidade pública listadas na BM\&FBovespa.

Conforme Bontempo (1997), a teoria dos jogos é um instrumento matemático, mas que vem sendo estudado de maneira intensa também nas aplicações de teoria econômica, bem como em outras áreas do conhecimento, como a contabilidade. No que tange a sua aplicação, destaca-se o estudo de Brandt, Fischer, Harrenstein e Shoham (2009), que utilizou a teoria dos jogos para determinar o ponto de equilíbrio de Nash entre o desempenho econômico e o desempenho social das empresas.

A análise do ponto de equilíbrio verifica as estratégias que as empresas deveriam assumir para alcançar os ganhos máximos com a minimização de suas perdas, tanto no desempenho econômico quanto no desempenho social de maneira simultânea. A delimitação de um ponto de equilíbrio permite às empresas atingir em um dado momento tanto ganhos máximos no desempenho econômico quanto no desempenho social, de modo a não ocasionar perdas a nenhum dos jogadores (Nash, 1950, 1951; Simonsen, 1994; Fiani, 2004; Lins \& Calôba, 2006).

No âmbito da análise do desempenho social, verifica-se ainda um grande número de estudos inconclusivos acerca dessa relação (Arlow \& Gannon, 1982; Preston \& O'Bannon, 1997). Aupperle et al. (1985), Ullmann (1985) e Arlow e Gannon (1992) efetuaram importantes descobertas, porém a discussão da relação do desempenho econômico-financeiro e desempenho social ainda não está completamente consolidada. Verifica-se uma diminuição nos estudos que apontam uma relação negativa entre o desempenho 
econômico e o social. Com os resultados apontados por Ullmann (1985) e Griffin e Mahon (1997), confirma-se a afirmação anteriormente emitida por Cochran e Wood (1984, segundo os quais, os pesquisadores não chegaram a um consenso sobre a relação entre essas variáveis, ou ainda não existe uma relação clara entre o desempenho econômico e social, sendo esta uma importante discussão para a gestão empresarial.

\section{TEORIA DOS JOGOS}

O princípio da teoria dos jogos, considerando-se sua desvinculação direta da teoria das probabilidades, ocorreu em meados do século $X X$, quando o matemático John Von Neumann introduziu a perspectiva de resolução de conflitos de interesse por meio da matemática (Souza, 2003). Porém ela tomou impulso a partir de 1944, quando Von Neumann e Oskar Morgenstern (1953) lançaram o livro Theory of games and economic behavior.

Conforme Fiani (2004), a teoria dos jogos consiste em um método aplicado ao processo de tomada de decisão quando dois ou mais agentes interagem entre si. Considerando a forma de interação entre jogadores, existem diferentes abordagens dentro da teoria dos jogos, como os jogos não cooperativos e os jogos cooperativos. Para a resolução de um jogo não cooperativo, que presume a existência de disputa entre os jogadores não possibilitando acordos entre eles, faz-se necessário, primeiramente, uma matriz com os ganhos e perdas dos jogadores. Assim, o Jogador A escolhe suas estratégias conforme dispostas nas linhas $(m)$, enquanto o Jogador $\mathrm{B}$ efetua suas escolhas baseado nos ganhos apresentados nas colunas ( $n$ ) (Lins \& Calôba, 2006).

Os jogos de soma zero consideram que a matriz de ganhos e perdas do Jogador A é oposta à matriz de ganhos e perdas do Jogador B. Entretanto, costumeiramente os ganhos e perdas entre os jogadores não são simétricos. Dessa forma, percebendo que o modelo utilizado gerará matrizes não opostas, ou seja, o modelo utilizado é de soma diferente de zero, será necessária a avaliação dos resultados por meio de jogos bimatriciais, recaindo-se na análise do ponto de equilíbrio de Nash. 
Na matriz de jogo para o modelo de equilíbrio de Nash, os jogadores são identificados como indicadores sociais (Jogador A) e indicadores econômicos (Jogador B). Nesse caso, considera-se uma matriz de pares ordenados refletidos em ganhos de $\mathrm{A}\left(a_{i j}\right)$ e ganhos de $\mathrm{B}\left(b_{j i}\right)$, conforme apresentado na matriz da Tabela 1.

Tabela 1: Matriz do jogo

\begin{tabular}{c|c|c|c|cc}
\hline \multirow{2}{*}{} & \multicolumn{3}{|c}{ Jogador B (Indicadores Econômicos) } \\
\cline { 3 - 6 } & & Indicador & Indicador & $\ldots$ & Indicador \\
\multicolumn{2}{c|}{} & 1 & 2 & & $n$ \\
\hline \multirow{3}{*}{$\begin{array}{c}\text { Jogador A } \\
\text { Sociais) }\end{array}$} & Indicador 1 & $\left(a_{11} ; b_{11}\right)$ & $\left(a_{12} ; b_{21}\right)$ & $\ldots$ & $\left(a_{n} ; b_{n}\right)$ \\
\cline { 2 - 6 } & Indicador 2 & $\left(a_{21} ; b_{12}\right)$ & $\left(a_{22} ; b_{22}\right)$ & $\ldots$ & $\left(a_{n} ; b_{n}\right)$ \\
\cline { 2 - 6 } & $\vdots$ & $\vdots$ & $\vdots$ & $\ddots$ & $\vdots$ \\
\cline { 2 - 6 } & Indicador & $\left(a_{m} ; b_{m}\right)$ & $\left(a_{m} ; b_{m}\right)$ & $\ldots$ & $\left(a_{m n} ; b_{m n}\right)$ \\
\hline
\end{tabular}

Fonte: Elaboração própria

A solução do jogo consiste em identificar o par ordenado cujo ganho seja máximo entre as alternativas e a perda seja mínima entre as estratégias do oponente (Simonsen, 1994). Os valores para identificar o ponto de equilíbrio foram determinados por meio de Regressão Linear Múltipla. A partir, desse método, utilizam-se os valores dos Coeficientes Beta Padronizados (Beta Standarized).

A utilização dos valores dos Coeficientes Beta Padronizados é abordada por Maroco (2003, p. 391) ao inferir que "[...] a simples comparação dos coeficientes de regressão para avaliar a importância de cada variável independente no modelo não é válida". Justifica-se, a utilização desses valores dos Coeficientes Beta Padronizados, pois as variáveis dependentes apresentam diferentes magnitudes. Dessa forma, "para que a importância de cada variável no modelo possa ser comparada, é necessário usar as variáveis standardizadas (por exemplo, $Z_{i j}=\left(X_{i j}-\bar{X}_{l}\right) / S_{X_{i}}^{\prime}$ ) no ajustamento do modelo ou então standardizar os coeficientes de regressão" (Maroco, 2003, p. 391). 
Desse modo, para os fins deste estudo, consideram-se apenas os valores de Beta Standard, sem se considerarem a eficácia do modelo e a significância das variáveis, pois objetiva-se apenas averiguar qual é a relação existente entre os indicadores e não os níveis de significância.

Nesse sentido, o modelo construído apresenta-se no formato descrito a seguir.

Sendo $X_{i}$ variável dependente representando os $i$-ésimos indicadores sociais, $Y_{i}$ variável independente representativa dos $j$-ésimos indicadores econômicos, há a formação da seguinte equação:

$$
\mathrm{X}_{\mathrm{i}}=a_{0 \mathrm{i}}+a_{1 \mathrm{i}} Y_{1}+a_{2 \mathrm{i}} Y_{2}+\cdots+a_{\mathrm{ni}} Y_{n}+\varepsilon_{i}
$$

com $i=1, \cdots, m$.

Assim são estabelecidos os Coeficientes Normalizados da equação $\alpha_{1 \mathrm{i}}, \alpha_{\mathrm{i} 2}, \cdots, \alpha_{\mathrm{ni}}$, da seguinte forma:

$$
\alpha_{\mathrm{ij}}=a_{\mathrm{ij}}\left(\frac{s_{Y_{j}}}{s_{X_{i}}}\right)
$$

Esses coeficientes $\alpha_{i j}$ representam os ganhos dos indicadores sociais $X_{i}$ (estratégias) por parte do Jogador $\mathrm{A}$, tendo em vista as $Y_{j}$ estratégias do jogador $B$.

De forma semelhante, para os indicadores econômicos, foram consideradas as variáveis $Y_{j}$ como as variáveis dependentes do modelo que representa os $j$-ésimos indicadores econômicos, bem como $X_{i}$ a variável independente, representando os $i$-ésimos indicadores sociais das empresas analisadas. Tem-se assim a equação:

$$
\mathrm{Y}_{\mathrm{j}}=b_{0 \mathrm{j}}+b_{1 \mathrm{j}} X_{1}+b_{2 \mathrm{j}} X_{2}+\cdots+b_{\mathrm{mj}} X_{m}+\varepsilon_{j}
$$

$\operatorname{com} j=1, \cdots, n$.

É possível obter os Coeficientes Normalizados da equação $\beta_{1 \mathrm{j}}, \beta_{2 \mathrm{j}}, \cdots, \beta_{\mathrm{nj}}$ , calculados como:

$$
\beta_{\mathrm{ij}}=b_{\mathrm{ij}}\left(\frac{s_{X_{i}}}{s_{Y_{j}}}\right)
$$

De forma análoga ao que foi considerado para as estratégias de $X_{i}$, as regressões identificam o valor de pagamento para $Y_{j}$ caso o jogador $\mathrm{B}$ o escolha como estratégia (Indicadores Econômicos), para todas as opções (Indicadores Sociais) do Jogador A. 
A matriz de comparação, foi adaptada da Tabela 1, e é construída conforme a Tabela 2.

Tabela 2: Matriz de comparação

\begin{tabular}{c|c|c|c|cc}
\hline \multirow{2}{*}{} & \multicolumn{4}{|c}{ Jogador B (Indicadores Econômicos) } \\
\cline { 3 - 6 } & $Y_{1}$ & $Y_{2}$ & $\cdots$ & $Y_{\mathrm{n}}$ \\
\hline Jogador A & $X_{1}$ & $\left(\alpha_{11} ; \beta_{11}\right)$ & $\left(\alpha_{12} ; \beta_{21}\right)$ & $\cdots$ & $\left(\alpha_{n} ; \beta_{n}\right)$ \\
(Indicadores & $X_{2}$ & $\left(\alpha_{21} ; \beta_{12}\right)$ & $\left(\alpha_{22} ; \beta_{22}\right)$ & $\ldots$ & $\left(\alpha_{n} ; \beta_{n}\right)$ \\
Sociais) & $\vdots$ & $\vdots$ & $\vdots$ & $\ddots$ & $\vdots$ \\
& $X_{\mathrm{m}}$ & $\left(\alpha_{m} ; \beta_{m}\right)$ & $\left(\alpha_{m} ; \beta_{m}\right)$ & $\ldots$ & $\left(\alpha_{m n} ; \beta_{m n}\right)$ \\
\hline
\end{tabular}

Fonte: Elaboração própria

Para determinar o ponto de equilíbrio de Nash em um jogo bimatricial, Simonsen (1994, p. 414) salienta que se deve identificar "[...] uma casa onde o ganho de A seja máximo na coluna e o de B máximo na linha".

Salienta-se que para a identificação dos pontos de equilíbrio observaram-se os sinais (negativos ou positivos) dos Coeficientes Beta obtidos pelas Regressões Lineares.

Desse modo, procura-se evidenciar quais as estratégias (indicadores) que as empresas deveriam assumir para atingir um equilíbrio entre o desempenho econômico e social. Nash $(1950 ; 1951)$ descreve que, tal como os jogos de soma zero, qualquer jogo bimatricial deve considerar a possibilidade de estratégias puras, quando há um ponto de equilíbrio de Nash, ou de estratégias mistas, quando não há um ponto específico para que a condição seja atendida. Considerando essa determinação, admitiram-se os sistemas e observa-se apenas a existência ou não de equilíbrio de Nash para estratégias puras.

Com base nessa afirmativa, formula-se a hipótese deste estudo, vinculada ao problema a ser investigado, sendo assim exposto: 
$\mathbf{H}_{\mathbf{0}}$ : Não há um ponto de equilíbrio de Nash no jogo bimatricial de indicadores econômicos e indicadores sociais das empresas do setor de Utilidade Pública.

Se a hipótese nula $\mathrm{H}_{0 b}$ não se confirmar, admitir-se-á a hipótese $\mathrm{H}_{1}$, que é dada nos seguintes termos:

H: Há ao menos um ponto de equilíbrio de Nash no jogo bimatricial de indicadores econômicos e indicadores sociais das empresas do setor de Utilidade Pública.

Nessa concepção, Simonsen (1994) corrobora os momentos em que possa ocorrer a presença de mais um ponto de equilíbrio, podendo admitir estratégias mistas neste caso para obter-se o melhor ponto de equilíbrio.

\section{METODOLOGIA}

Nesta pesquisa, o objetivo é analisar a relação entre indicadores de desempenho econômico e social das empresas listadas no setor de Utilidade Pública da BM\&FBovespa mediante aplicação da teoria dos jogos para jogos bimatriciais.

Como população, delimitaram-se todas as empresas pertencentes ao setor de Utilidade Pública - que compreende $30 \%$ das empresas não financeiras que realizam negociações na BM\&FBovespa -, perfazendo um total de 75 organizações. Essa delimitação da população abarca o setor mais representativo de janeiro de 2014, conforme a classificação setorial da BM\&FBovespa.

Portanto verifica-se que nem todas as empresas disponibilizavam as demonstrações contábeis consolidadas, bem como as informações necessárias para o cálculo dos indicadores de desempenho para os períodos em questão (2009 a 2011). Dessa forma, efetuou-se a exclusão dessas empresas, sendo a amostra final composta por 26 empresas do setor de Utilidade Pública.

A utilização dos dados para a realização da análise consiste em um conjunto de indicadores de maior relevância para a mensuração do desempenho econômico e social das empresas. Para caracterizar a situação econômica das empresas, utilizaram-se os seguintes indicadores: Retorno do Ativo (ROA), Retorno do Patrimônio Líquido (ROE), Margem Líquida (ML), 
Lucro Por Ação (LPA) e Índice Preço/Lucro (P/L). A utilização desses indicadores está pautada em Brigham e Houston (1999), Schrickel (1999), Brigham, Gapenski e Ehrhardt (2001), Salazar e Benedicto (2004), Savytzky (2005), Brigham e Ehrhardt (2006), Assaf Neto (2009), Hoji (2012) e Silva (2012).

O desempenho social das empresas foi caracterizado sob a ótica da Demonstração do Valor Adicionado, devido à grande utilidade social dessa demonstração. Os indicadores utilizados foram: Gastos com Pessoal (GP), Gastos com Tributos (GT), Quociente do Valor Adicionado (QVA), Taxa de Variação do VA Bruto (TVAB), Taxa de Variação Total do VA Bruto (TVT) e o Potencial do Ativo em Gerar Riqueza (PAGR). Esses indicadores apresentam suporte teórico nos trabalhos de Bao e Bao (1998), Haller e Stolowy (1998), Santos (1999), Cosenza (2003), Aswegen, Steyn e Hamman (2005), Santos (2007), Nandi (2011).

A utilização da teoria dos jogos justifica-se pelo objetivo proposto nesta pesquisa, impossibilitando a aplicação de outras técnicas como dados em painel, por exemplo, visto que a análise a ser efetuada é transversal.

Os resultados para identificação do ponto de equilíbrio serão definidos mediante regressões lineares para determinar os valores dos Betas estandardizados, efetuadas por meio do software SPSS. As regressões lineares serão efetuadas da seguinte forma: primeiramente realiza-se uma regressão entre um indicador social (variável dependente) com todos os indicadores econômicos (variáveis independentes). Dessa forma, os coeficientes padronizados de Beta encontrados estarão dispostos nas linhas. $\mathrm{Na}$ sequência, efetuam-se os mesmos procedimentos, por sua vez para os indicadores econômicos que serão considerados as variáveis dependentes e os indicadores sociais as variáveis independentes. Dessa forma, os coeficientes encontrados serão dispostos nas colunas. Nesse contexto, para determinar o ponto de equilíbrio de Nash em um jogo bimatricial, Simonsen (1994, p. 414) salienta que se deve identificar "[...] onde o ganho de A seja máximo na coluna e o de B máximo na linha". Dessa forma, é identificado o ponto de equilíbrio do jogo, devendo as empresas posicionar suas estratégias para esses indicadores a fim de alcançar um equilíbrio entre o desempenho econômico e o social. 


\section{ANÁLISE DOS DADOS}

Busca-se nesta seção descrever a identificação dos pontos de equilíbrio de Nash de um jogo bimatricial entre indicadores econômicos e sociais.

Para determinar o ponto de equilíbrio, definiram-se os valores com base na Regressão Linear Simples, considerando-se apenas os valores dos coeficientes padronizados Beta, pois eles indicam a relação entre as variáveis independentes e a variável dependente. Na Tabela 3, apresentam-se os resultados das regressões para o período de 2009 a 2011, assumindo-se como variáveis dependentes os indicadores econômicos e por variáveis independentes os indicadores sociais. Ou seja, primeira regressão com ROA na variável dependente e todos os indicadores sociais na variável independente, e assim sucessivamente.

Tabela 03: Resultados das regressões lineares

\section{9}

\begin{tabular}{l|r|r|r|r|r|r|r|r|r|r}
\hline & \multicolumn{2}{|c}{ ROA } & \multicolumn{2}{c|}{ ROE } & \multicolumn{2}{c}{ ML } & \multicolumn{3}{c}{ LPA } & \multicolumn{3}{c}{ PL } \\
\hline & $\begin{array}{l}\text { Beta } \\
\text { Pad. }\end{array}$ & Sig. & $\begin{array}{l}\text { Beta } \\
\text { Pad. }\end{array}$ & Sig. & $\begin{array}{l}\text { Beta } \\
\text { Pad. }\end{array}$ & Sig. & $\begin{array}{l}\text { Beta } \\
\text { Pad. }\end{array}$ & Sig. & $\begin{array}{l}\text { Beta } \\
\text { Pad. }\end{array}$ & Sig. \\
\hline Constant & &, 246 & &, 766 & &, 295 & &, 967 &, 000 \\
\hline GP &,- 201 &, 424 &, 050 &, 856 &,- 247 &, 379 &, 100 &, 699 &, 134 &, 195 \\
\hline GT &, 281 &, 381 &,- 198 &, 575 &, 313 &, 379 &,- 113 &, 731 &, 921 &, 000 \\
\hline QVA &,- 311 &, 168 &,- 135 &, 581 &,- 050 &, 837 &,- 207 &, 365 &, 233 &, 016 \\
\hline TVAB &,- 041 &, 871 &, 013 &, 962 &,- 057 &, 838 &,- 094 &, 718 &, 203 &, 059 \\
\hline TVT &,- 001 &, 996 &, 077 &, 800 &,- 103 &, 735 &, 074 &, 795 &, 056 &, 617 \\
\hline PAGR &, 062 &, 843 &, 275 &, 433 &,- 217 &, 537 &, 394 &, 233 &,- 109 &, 396 \\
\hline $\mathbf{R}^{2}$ & &, 239 & &, 067 & &, 063 & &, 194 & &, 875 \\
\hline
\end{tabular}


Continuação

2010

\begin{tabular}{l|r|l|l|l|l|l|l|l|l|l}
\hline & \multicolumn{2}{|c|}{ ROA } & \multicolumn{2}{c|}{ ROE } & \multicolumn{2}{c|}{ ML } & \multicolumn{2}{c|}{ LPA } & \multicolumn{2}{c}{ PL } \\
\hline & $\begin{array}{l}\text { Beta } \\
\text { Pad. }\end{array}$ & Sig. & $\begin{array}{l}\text { Beta } \\
\text { Pad. }\end{array}$ & Sig. & $\begin{array}{l}\text { Beta } \\
\text { Pad. }\end{array}$ & Sig. & $\begin{array}{l}\text { Beta } \\
\text { Pad. }\end{array}$ & Sig. & $\begin{array}{l}\text { Beta } \\
\text { Pad. }\end{array}$ & Sig. \\
\hline Constant & &, 190 & &, 687 & &, 319 & &, 165 & &, 000 \\
\hline GP &, 153 &, 593 &,- 306 &, 346 &,- 222 &, 495 &, 329 &, 276 &,- 383 &, 088 \\
\hline GT &,- 875 &, 035 &, 139 &, 753 &,- 083 &, 852 &,- 582 &, 164 &, 735 &, 021 \\
\hline QVA &,- 651 &, 019 &,- 249 &, 398 &,- 211 &, 475 &,- 223 &, 413 &, 275 &, 172 \\
\hline TVAB &, 015 &, 948 &,- 053 &, 842 &, 358 &, 186 &,- 315 &, 205 &,- 426 &, 024 \\
\hline TVT &,- 020 &, 935 &, 277 &, 323 &, 207 &, 460 &,- 121 &, 637 &,- 479 &, 017 \\
\hline PAGR &, 726 &, 081 &,- 182 &, 687 &,- 199 &, 662 &, 790 &, 070 &,- 656 &, 041 \\
\hline$R^{2}$ & \multicolumn{1}{r}{, 337} & &, 154 & &, 143 & &, 275 & &, 618 \\
\hline
\end{tabular}

\section{1}

\begin{tabular}{l|c|c|c|c|c|c|c|c|c|c}
\hline & \multicolumn{2}{|c|}{ ROA } & \multicolumn{2}{c|}{ ROE } & \multicolumn{2}{c|}{ ML } & \multicolumn{2}{c|}{ LPA } & \multicolumn{2}{c}{ PL } \\
\hline & $\begin{array}{l}\text { Beta } \\
\text { Pad. }\end{array}$ & Sig. & $\begin{array}{l}\text { Beta } \\
\text { Pad. }\end{array}$ & Sig. & $\begin{array}{l}\text { Beta } \\
\text { Pad. }\end{array}$ & Sig. & $\begin{array}{l}\text { Beta } \\
\text { Pad. }\end{array}$ & Sig. & $\begin{array}{l}\text { Beta } \\
\text { Pad. }\end{array}$ & Sig. \\
\hline Constant & &, 298 & &, 391 & &, 799 & &, 638 & &, 895 \\
\hline GP &,- 117 &, 784 &,- 067 &, 866 &,- 455 &, 326 &, 154 &, 736 &, 444 &, 312 \\
\hline GT &,- 379 &, 398 &,- 211 &, 616 &, 373 &, 441 &,- 364 &, 450 &,- 448 &, 330 \\
\hline QVA &,- 350 &, 226 &,- 537 &, 055 &, 075 &, 806 &,- 261 &, 397 &,- 138 &, 635 \\
\hline TVAB &,- 071 &, 793 &,- 108 &, 673 &,- 065 &, 823 &,- 062 &, 831 &, 034 &, 901 \\
\hline TVT &, 138 &, 664 &, 111 &, 709 &, 111 &, 746 &, 067 &, 843 &,- 142 &, 662 \\
\hline PAGR &, 065 &, 888 &, 169 &, 696 &,- 403 &, 421 &, 147 &, 766 &, 779 &, 109 \\
\hline R & &, 193 & &, 285 & &, 059 & &, 066 & &, 157 \\
\hline
\end{tabular}

Fonte: Dados da pesquisa

A princípio, como se buscou analisar a possível existência de influência entre as variáveis independentes e a variável dependente, não foi levado em consideração o coeficiente de significância dos coeficientes Beta Padronizados. Para confirmação e prova de robustez, esse coeficiente deveria ser levado em consideração, entretanto neste trabalho busca-se mais 
descrever o modelo de equilíbrio para fins gerenciais e não para fins de inferência estatística dos resultados.

$\mathrm{Na}$ Tabela 4, apresentam-se os resultados das regressões para o período de 2009 a 2011, assumindo-se como variáveis dependentes os indicadores sociais e como variáveis independentes os indicadores econômicos. Ou seja, na primeira regressão, assume-se GP como variável dependente e todos os indicadores como variáveis independentes, e assim sucessivamente.

Tabela 04: Resultados das regressões lineares

\section{9}

\begin{tabular}{c|c|c|c|c|c|c|c|c|c|c|c|c}
\hline & \multicolumn{2}{|c|}{ GP } & \multicolumn{2}{c|}{ GT } & \multicolumn{2}{c|}{ QVA } & \multicolumn{2}{c|}{ TVAB } & \multicolumn{2}{c}{ TVT } & \multicolumn{2}{c}{ PAGR } \\
\hline & $\begin{array}{c}\text { Beta } \\
\text { Pad }\end{array}$ & Sig. & $\begin{array}{c}\text { Beta } \\
\text { Pad }\end{array}$ & Sig. & $\begin{array}{c}\text { Beta } \\
\text { Pad }\end{array}$ & Sig. & $\begin{array}{c}\text { Beta } \\
\text { Pad }\end{array}$ & Sig. & $\begin{array}{c}\text { Beta } \\
\text { Pad }\end{array}$ & Sig. & $\begin{array}{c}\text { Beta } \\
\text { Pad }\end{array}$ & Sig. \\
\hline Const & &, 008 & &, 000 & &, 005 & &, 000 & &, 000 & &, 000 \\
\hline ROE &,- 348 &, 266 &, 105 &, 519 &,- 628 &, 049 &,- 238 &, 472 &, 039 &, 902 &, 148 &, 620 \\
\hline ROA &, 108 &, 744 &,- 290 &, 108 &, 446 &, 183 &, 354 &, 324 &, 028 &, 935 &,- 206 &, 520 \\
\hline ML &,- 035 &, 869 &, 041 &, 714 &, 059 &, 776 &,- 053 &, 814 &,- 098 &, 653 &,- 040 &, 845 \\
\hline LPA &, 055 &, 844 &, 262 &, 084 &,- 270 &, 329 &,- 301 &, 315 &, 089 &, 755 &, 374 &, 171 \\
\hline PL &, 485 &, 034 &, 813 &, 000 &, 274 &, 208 &, 274 &, 244 &, 371 &, 107 &, 338 &, 116 \\
\hline $\mathbf{R}^{\mathbf{2}}$ & \multicolumn{2}{|c|}{, 221} & \multicolumn{2}{|c|}{, 785} & \multicolumn{2}{|c|}{, 244} & \multicolumn{2}{c}{, 115} &, 180 & &, 282 \\
\hline
\end{tabular}

\section{0}

\begin{tabular}{|c|c|c|c|c|c|c|c|c|c|c|c|c|}
\hline & \multicolumn{2}{|c|}{ GP } & \multicolumn{2}{|c|}{ GT } & \multicolumn{2}{|c|}{ QVA } & \multicolumn{2}{|c|}{ TVAB } & \multicolumn{2}{|c|}{ TVT } & \multicolumn{2}{|c|}{ PAGR } \\
\hline & $\begin{array}{l}\text { Beta } \\
\text { Pad }\end{array}$ & Sig. & $\begin{array}{l}\text { Beta } \\
\text { Pad }\end{array}$ & Sig. & $\begin{array}{l}\text { Beta } \\
\text { Pad }\end{array}$ & Sig. & $\begin{array}{l}\text { Beta } \\
\text { Pad }\end{array}$ & Sig. & $\begin{array}{l}\text { Beta } \\
\text { Pad }\end{array}$ & Sig. & $\begin{array}{l}\text { Beta } \\
\text { Pad }\end{array}$ & Sig. \\
\hline Const & & ,005 & & ,000 & & ,005 & & ,000 & & ,000 & & ,000 \\
\hline ROA &,- 204 & ,609 &,- 434 & ,273 &,- 156 & 669 & 298 & ,319 &,- 476 & ,119 &,- 328 & ,382 \\
\hline ROE &,- 089 & 791 & ,403 & ,228 &,- 463 & 142 &,- 588 & ,026 & ,338 & 185 & 379 & ,234 \\
\hline$M L$ &,- 093 & ,694 & , 151 &, 513 &,- 029 & ,894 & 179 & ,310 & 177 & ,319 & , 126 & ,568 \\
\hline LPA & 103 & ,826 & ,430 & ,355 &,- 286 & ,509 &,- 903 & ,016 & 486 & ,174 & ,517 & ,246 \\
\hline PL &,- 202 & ,368 &,- 155 & ,478 &,- 183 & ,373 &,- 453 & 011 &,- 633 & ,001 &,- 327 & , 125 \\
\hline $\mathbf{R}^{2}$ & \multicolumn{2}{|c|}{ 082, } & \multicolumn{2}{|c|}{120} & \multicolumn{2}{|c|}{,231 } & \multicolumn{2}{|c|}{,496 } & \multicolumn{2}{|c|}{,492 } & \multicolumn{2}{|c|}{,203 } \\
\hline
\end{tabular}


Continuação

\section{1}

\begin{tabular}{|c|c|c|c|c|c|c|c|c|c|c|c|c|}
\hline & \multicolumn{2}{|c|}{ GP } & \multicolumn{2}{|c|}{ GT } & \multicolumn{2}{|c|}{ QVA } & \multicolumn{2}{|c|}{ TVAB } & \multicolumn{2}{|c|}{ TVT } & \multicolumn{2}{|c|}{ PAGR } \\
\hline & $\begin{array}{l}\text { Beta } \\
\text { Pad }\end{array}$ & Sig. & $\begin{array}{l}\text { Beta } \\
\text { Pad }\end{array}$ & Sig. & $\begin{array}{l}\text { Beta } \\
\text { Pad }\end{array}$ & Sig. & $\begin{array}{l}\text { Beta } \\
\text { Pad }\end{array}$ & Sig. & $\begin{array}{l}\text { Beta } \\
\text { Pad }\end{array}$ & Sig. & $\begin{array}{l}\text { Beta } \\
\text { Pad }\end{array}$ & Sig. \\
\hline Const & & ,005 & & ,000 & & ,000 & & ,000 & & ,000 & & ,000 \\
\hline ROE &,- 585 & 203 &,- 785 & ,089 & ,566 & , 166 & ,216 & 653 &,- 538 & 243 &,- 147 & ,745 \\
\hline ROA & ,112 & 728 & 480 & 140 &,- 786 & 011 &,- 159 & ,643 & ,515 & 122 & ,249 & ,440 \\
\hline$M L$ &,- 015 & 945 & 154 & ,485 &,- 111 & ,574 &,- 085 & ,718 & 128 & ,569 & ,055 & 803 \\
\hline LPA & ,386 & ,356 & ,527 & 206 &,- 672 & ,078 &,- 201 & ,649 & ,516 & ,223 & 102 & 805 \\
\hline $\mathrm{PL}$ &,- 144 & ,503 & ,046 & 828 & ,005 & ,980 &,- 050 & 825 & ,079 & ,713 & ,334 & 130 \\
\hline $\mathbf{R}^{2}$ & \multicolumn{2}{|c|}{, 133} & \multicolumn{2}{|c|}{ 155 } & \multicolumn{2}{|c|}{,322 } & \multicolumn{2}{|c|}{,018 } & \multicolumn{2}{|c|}{124} & \multicolumn{2}{|c|}{134} \\
\hline
\end{tabular}

Fonte: Dados da pesquisa

Realizadas as regressões lineares, na sequência, determina-se o ganho máximo disposto na coluna para o Jogador A (indicadores econômicos) e o ganho máximo de disposto na linha para o Jogador B (indicadores sociais) conforme elencado por Simonsen (1994). Dessa maneira, encontra-se o ponto de equilíbrio de Nash para um jogo bimatricial, de modo que as empresas posicionem suas estratégias para tais indicadores a fim de alcançar um equilíbrio entre o desempenho econômico e o desempenho social.

Apresentam-se na Tabela 5 os resultados para o ponto de equilíbrio de Nash para o período de 2009 a 2011.

Tabela 5: Ponto de equilíbrio de Nash do setor de Utilidade Pública

\section{9}

\begin{tabular}{l|l|l|l|l|l|l}
\hline & ROA & ROE & ML & LPA & P/L & Max $\boldsymbol{b}_{\boldsymbol{i j}}$ \\
\hline GP & $(-0,348 ;-0,201)$ & $(0,108 ; 0,050)$ & $(-0,035 ;-0,247)$ & $(0,055 ; 0,100)$ & $(0,485 ; \mathbf{0 , 1 3 4 )}$ & $\mathbf{0 , 1 3 4}$ \\
\hline GT & $(0,105 ; 0,281)$ & $(-0,290 ;-0,198)$ & $(0,041 ; 0,313)$ & $(0,262 ;-0,113)$ & $(\mathbf{0 , 8 1 3 ; 0 , 9 2 1 )}$ & $\mathbf{0 , 9 2 1}$ \\
\hline QVA & $(-0,628 ;-0,311)$ & $(\mathbf{0 , 4 4 6 ; - 0 , 1 3 5 )}$ & $(\mathbf{0 , 0 5 9} ;-0,050)$ & $(-0,270 ;-0,207)$ & $(0,274 ; \mathbf{0 , 2 3 3 )}$ & $\mathbf{0 , 2 3 3}$ \\
\hline TVAB & $(-0,238 ;-0,041)$ & $(0,354 ; 0,013)$ & $(-0,053 ;-0,057)$ & $(-0,301 ;-0,094)$ & $(0,274 ; \mathbf{0 , 2 0 3})$ & $\mathbf{0 , 2 0 3}$ \\
\hline
\end{tabular}


Continuação

\begin{tabular}{l|l|l|l|l|l|l}
\hline TVT & $(0,039 ;-0,001)$ & $(0,028 ; \mathbf{0 , 0 7 7})$ & $(-0,098 ;-0,103)$ & $(0,089 ; 0,074)$ & $(0,371 ; 0,056)$ & $\mathbf{0 , 0 7 7}$ \\
\hline PAGR & $(\mathbf{0 , 1 4 8 ; 0 , 0 6 2 )}$ & $(-0,206 ; 0,275)$ & $(-0,040 ;-0,217)$ & $(\mathbf{0 , 3 7 4 ; 0 , 3 9 4 )}$ & $(0,338 ;-0,109)$ & $\mathbf{0 , 3 9 4}$ \\
\hline Max & & & & & & \\
$\boldsymbol{a}_{i j}$ & $\mathbf{0 , 1 4 8}$ & $\mathbf{0 , 4 4 6}$ & $\mathbf{0 , 0 5 9}$ & $\mathbf{0 , 3 7 4}$ & $\mathbf{0 , 8 1 3}$ & \\
\hline
\end{tabular}

2010

\begin{tabular}{|c|c|c|c|c|c|c|}
\hline & ROA & ROE & $M L$ & LPA & $P / L$ & $\operatorname{Max} b_{i j}$ \\
\hline GP & $(-0,204 ; 0,153)$ & $(-0,089 ;-0,306)$ & $(-0,093 ;-0,222)$ & $(0,103 ; 0,329)$ & $(-0,202 ;-0,383)$ & 0,329 \\
\hline GT & $(-0,434 ;-0,875)$ & $(0,403 ; 0,139)$ & $(0,151 ;-0,083)$ & $(0,430 ;-0,582)$ & $(-0,155 ; 0,735)$ & 0,735 \\
\hline QVA & $(-0,156 ;-0,651)$ & $(-0,463 ;-0,249)$ & $(-0,029 ;-0,211)$ & $(-0,286 ;-0,223)$ & $(-0,183 ; \mathbf{0}, 275)$ & 0,275 \\
\hline TVAB & $(\mathbf{0 , 2 9 8} ; 0,015)$ & $(-0,588 ;-0,053)$ & $(0,179 ; 0,358)$ & $(-0,903 ;-0,315)$ & $(-0,453 ;-0,426)$ & 0,358 \\
\hline TVT & $(-0,476 ;-0,020)$ & $(0,338 ; \mathbf{0 , 2 7 7})$ & $(0,177 ; 0,207)$ & $(0,486 ;-0,121)$ & $(-0,633 ;-0,479)$ & 0,277 \\
\hline PAGR & $(-0,328 ; 0,726)$ & $(0,379 ;-0,182)$ & $(0,126 ;-0,199)$ & $(0,517 ; 0,790)$ & $(-0,327 ;-0,656)$ & 0,790 \\
\hline $\begin{array}{l}\text { Max } \\
a_{i j}\end{array}$ & 0,298 & 0,403 & 0,179 & 0,517 & $-0,155$ & \\
\hline
\end{tabular}

2011

\begin{tabular}{|c|c|c|c|c|c|c|}
\hline & ROA & ROE & $M L$ & LPA & $P / L$ & $\operatorname{Max} b_{i j}$ \\
\hline GP & $(-0,585 ;-0,117)$ & $(0,112 ;-0,067)$ & $(-0,015 ;-0,455)$ & $(0,386 ; 0,154)$ & $(-0,144 ; \mathbf{0}, \mathbf{4 4 4})$ & 0,444 \\
\hline GT & $(-0,785 ;-0,379)$ & $(0,480 ;-0,211)$ & $(0,154 ; 0,373)$ & $(\mathbf{0 , 5 2 7} ;-0,364)$ & $(0,046 ;-0,448)$ & 0,373 \\
\hline QVA & $(\mathbf{0 , 5 6 6} ;-0,350)$ & $(-0,786 ;-0,537)$ & $(-0,111 ; \mathbf{0 , 0 7 5})$ & $(-0,672 ;-0,261)$ & $(0,005 ;-0,138)$ & 0,075 \\
\hline TVAB & $(0,216 ;-0,071)$ & $(-0,159 ;-0,108)$ & $(-0,085 ;-0,065)$ & $(-0,201 ;-0,062)$ & $(-0,050 ; \mathbf{0 , 0 3 4 )}$ & 0,034 \\
\hline TVT & $(-0,538 ; \mathbf{0 , 1 3 8})$ & $(0,515 ; 0,111)$ & $(0,128 ; 0,111)$ & $(0,516 ; 0,067)$ & $(0,079 ;-0,142)$ & 0,138 \\
\hline PAGR & $(-0,147 ; 0,065)$ & $(0,249 ; 0,169)$ & $(0,055 ;-0,403)$ & $(0,102 ; 0,147)$ & $(0,334 ; 0,779)$ & 0,779 \\
\hline $\begin{array}{l}\text { Max } \\
a_{i j}\end{array}$ & 0,566 & 0,515 & 0,154 & 0,527 & 0,334 & \\
\hline
\end{tabular}

Fonte: Dados da pesquisa

Conforme os dados apresentados na Tabela 5, a primeira combinação encontrada que induz a ganhos no desempenho econômico e no desempenho social de maneira conjunta é entre o indicador de Lucro por Ação com o Potencial do Ativo em Gerar Riqueza. Outra estratégia que gera ganhos 
comuns a indicadores econômicos e sociais adotada pelas empresas em 2009 é dada pela combinação do Índice Preço/Lucro com os Gastos com Tributos.

No período de 2010, identificaram-se três estratégias que acarretaram um equilíbrio para as duas formas de avaliação de desempenho analisadas na pesquisa. Infere-se que as empresas adotaram por estratégias a combinação da Margem Líquida com a Taxa de Variação do Valor Adicionado Bruto, o Lucro por Ação com o Potencial do Ativo em Gerar Riqueza e ainda o Índice Preço/Lucro em combinação com os Gastos com Tributos.

Para o período de 2011, as empresas do setor de Utilidade Pública adotaram duas combinações estratégicas que combinadas geraram ganhos para ambos os grupos de indicadores: Margem Líquida com os Gastos com Tributos e ainda o Índice Preço/Lucro com o Potencial do Ativo em Gerar Riqueza.

Constata-se que as empresas do setor de Utilidade Pública que utilizam por foco o indicador do Quociente do Valor Adicionado e da Taxa de Variação Total do Valor Adicionado, tendem a prejudicar todos os seus indicadores econômicos, assim como o Retorno sobre o Ativo e o Retorno sobre o Patrimônio Líquido tendem a prejudicar os indicadores sociais. Essa constatação é efetuada, pois esses indicadores não se encontram em nenhuma das estratégias adotadas pelas empresas e identificadas no ponto de equilíbrio de Nash.

Esses são os pontos de equilíbrio de Nash identificados nos jogos, para os quais as empresas podem posicionar suas estratégias, de modo a alcançar um equilíbrio entre o desempenho econômico e o desempenho social, no qual não se obtêm ganhos para satisfazer nem o primeiro conjunto de indicadores nem o segundo conjunto sem onerar nenhuma das duas categorias de informações.

Conforme os dados apresentados, é possível perceber que, para todos os períodos analisados, encontrou-se no mínimo um ponto de equilíbrio. É para esses indicadores que as empresas podem posicionar suas estratégias, para alcançar um equilíbrio entre o desempenho econômico e o social, sem causar desconforto a nenhum dos jogadores.

Esse achado implica a rejeição da hipótese nula $\left(\mathrm{H}_{0 b}\right)$ e a aceitação da hipótese $\left(\mathrm{H}_{1 b}\right)$, assumindo-se que há pelo menos um ponto de equilíbrio de 
Nash no jogo bimatricial de indicadores econômicos e indicadores sociais. Corrobora-se, dessa forma, a premissa estabelecida por Nash (1950; 1951) que definiu que qualquer jogo pautado em estratégias mistas apresenta um ponto de equilíbrio. Simonsen (1994) alerta para os momentos em que possa ocorre a identificação de mais de um ponto de equilíbrio no jogo. Dessa maneira, a combinação de estratégias dos indicadores econômicos com os indicadores sociais acarreta um equilíbrio dos desempenhos econômico e social das empresas.

\section{CONCLUSÃO}

Durante longos períodos de tempo, a grande preocupação das empresas era seu desempenho econômico e financeiro, porém, surgiu uma nova perspectiva cuja ênfase, para a análise de desempenho, não estava mais no lucro. Desse modo insere-se a discussão em torno da sustentabilidade empresarial, que está pautada em um equilíbrio econômico, social e ambiental para as empresas.

Diante dessa discussão, na presente pesquisa o objetivo é identificar o ponto de equilíbrio de Nash no jogo bimatricial de indicadores econômicos e indicadores sociais das empresas do setor de Utilidade Pública listadas na BM\&FBovespa. Realizou-se a pesquisa com uma amostra de 26 empresas. A escolha dos indicadores para a avaliação de desempenho econômico está pautada em indicadores de rentabilidade e de mercado; para a avaliação de desempenho social, utilizaram-se os indicadores oriundos da DVA (Demonstração do Valor Adicionado).

No modelo descrito, houve grande dificuldade na estimação dos valores para as matrizes de jogos. Estimar os ganhos entre os indicadores foi a grande dificuldade encontrada no trabalho. Por isso, utilizou-se para estimação dos parâmetros da matriz de jogo os parâmetros padronizados da regressão linear múltipla. A utilização dos coeficientes padronizados leva em consideração que os coeficientes estimados de uma regressão são influenciados diretamente pela magnitude ou grandeza da variável. Por outro lado, os coeficientes padronizados estão livres dessa implicação. Foi possível, 
assim, a comparação e, portanto, sua utilização na estimação dos pesos da matriz de jogos.

Nos resultados obtidos, destacou-se a existência de pelo menos um ponto de equilíbrio para os anos analisados, e em especial propuseram-se dois pontos de equilíbrio para 2009 e 2011, e três para 2010. Esse número maior de números favorece um possível processo de tomada de decisão por parte dos usuários dessa informação.

Destaca-se que o modelo proposto confirmou a hipótese da existência de pelo menos um ponto de equilíbrio, e assim a pesquisa consegue satisfazer seu objetivo de que é possível atender tanto aos aspectos econômicos quanto aos aspectos sociais das empresas quando se tomam como informações básicas os indicadores. Questionamentos que podem ser tratados com maior profundidade referem-se à importância do coeficiente de significância das regressões para o modelo, além de levar em consideração algum critério de obtenção do ponto de equilíbrio de Nash para estratégias mistas.

\section{REFERÊNCIAS}

Arlow, P., \& Gannon, M. J. (1982). Social responsiveness, corporate structure and economic performance. Academy of Management Review, $7(2), 235-241$.

Assaf Neto, A. (2009). Finanças corporativas e valor (4a ed.). São Paulo: Atlas.

Aswegen, N. V., Steyn, B. W., \& Hamman, W. D. (2005). Trends in the distribution of added value of listed industrial companies - 1990 to 2002. South African Journal of Business Management, 36(2), 85-94.

Aupperle, K. E., Carroll, A. B., \& Hatfield, J. D. (1985). An empirical examination of the relationship between corporate social responsibility and profitability. Academy of Management Journal, 28(2), 446-463.

Bao, B.-H., \& Bao, D.-H. (1998). Usefulness of value added and abnormal economic earnings: an empirical examination. Journal of Business Finance \& Accounting, 25(1-2), 251-264.

Baumgartner, R. J., \& Ebner, D. (2010, March-April). Corporate sustainability strategies: sustainability profiles and maturity levels. Sustainable Development, 18(2), 76-89.

Bontempo, M. T. (1997). Teoria dos jogos aplicada à contabilidade. Caderno de Estudos, 9(15), 35-40. 
Brandt,F., Fischer,F., Harrenstein, P., \& Shoham,Y. (2009). Ranking games. Artificial Intellingence, 173(2), 221-239.

Brigham, E. F., \& Ehrhardt, M. C. (2006). Administração financeira: teoria e prática. São Paulo: Pioneira Thomson Learning.

Brigham, E. F., Gapenski, L., \& Ehrhardt, M. (2001). Administração financeira: teoria e prática. São Paulo: Atlas.

Brigham, E., \& Houston, J. (1999). Fundamentos da moderna administração financeira. Rio de Janeiro: Campus.

Callado, A. L. C. (2010). Modelo de mensuração de sustentabilidade empresarial: uma aplicação em vinícolas localizadas na Serra Gaúcha. Tese de Doutorado em Agronegócios, Universidade Federal do Rio Grande do Sul, Porto Alegre, RS, Brasil.

Callado, A. L. C., \& Feinsterseifer, J. E. (2010). Mensuração de sustentabilidade através do Grid de Sustentabilidade Empresarial (GSE): um estudo no setor vinícola. Anais da Sociedade Brasileira de Economia, Administração e Sociologia Rural - SOBER, 48, Campo Grande, MS, Brasil.

Carroll, A. B. (1991). The pyramid of corporate social responsibility: toward the moral management of organizational stakeholders. Business Horizons, 34(4), 39-48.

Clarke-Sather, A. R., Hutchins, M. J., Zhang, Q., Gershenson, J. K., \& Sutherland, J. W. (2011). Development of social, environmental, and economic indicators for a small/medium enterprise. International Journal of Accounting and Informations Management, 19(3), 247-266.

Claro, P. B., Claro, D. P., \& Amâncio, R. (2008). Entendendo o conceito de sustentabilidade nas organizações. Revista de Administração, 43(4), 289300.

Cochran, P. L., \& Wood, R. A. (1984). Corporate social responsibility and financial performance. Academy of Management Journal, 27(1), 42-56.

Cosenza, J. P. (2003). A eficácia informativa da demonstração do valor adicionado. Revista Contabilidade \& Finanças, Edição comemorativa, 729.

Dyllick, T., \& Hockerts, K. (2002). Beyond the business case for corporate sustainability. Business Strategy and the environment, 11(2), 130-141.

Ebner, D., \& Baumgartner, R. J. (2006). The relationship between sustainable development and corporate social responsibility. Proceedings of Corporate Responsibility Research Conference, 2, Dublin, UK.

Fiani, R. (2004). Teoria dos jogos: para cursos de administração e economia. Rio de Janeiro: Elsevier. 
Griffin, J. J., \& Mahon, J. F. (1987). The corporate social performance and corporate financial performance debate: twenty-five years of incomparable research. Business \& Society, 36(1), 5-31.

Haller, A., \& Stolowy, H. (1998). Value added in financial accounting: a comparative study between Germany and France. Advances in International Accounting, 11(1), 23-51.

Hoji, M. (2012). Administração financeira e orçamentária: matemática financeira aplicada, estratégias financeiras, orçamento empresarial (10a ed.). São Paulo: Atlas.

Ioannou, I., \& Serafeim, G. (2012, August). The consequences of mandatory corporate sustainability reporting. Harvard Business School Research Working Paper No. 11-100.

Krajnc, D., \& Glavic, P. (2005). A model for integrated assessment of sustainable development. Resources, Conservation and Recycling, 43(2), 189-208.

Lee, K.-H., \& Saen, R. F. (2012). Measuring corporate sustainability management: a data envelopment analysis approach. International Journal of Production Economics, 140(1), 219-226.

Lins, M. P. E., \& Calôba, G. M. (2006). Programação linear: com aplicações em teoria dos jogos e avaliação de desempenho (data envelopment analysis). Rio de Janeiro: Interciência.

Lo, Shih-Fang. (2010, November-December). Performance evaluation for sustainable businnes a profitability and marketability framework. Corporate social responsibility and environmental management, 17(6), 311-319.

Maroco, J. (2003). Análise estatística: com utilização do SPSS (2a ed.). Lisboa: Sílabo.

Nandi, K. C. (2011). Performance measures: an application of value added statement. The IUP Journal of Operations Management, 10(3), 39-62.

Nash, J. (1950). Non-cooperative games. Dissertation, Doctor of Philosophy, Faculty of Princeton, New Jersey, USA.

Nash, J. (1951). Non-cooperative games. Annals of Mathematics, 54(2), 286-295.

Preston, L. E., \& O'Bannon, D. P. (1997). The corporate social-financial performance relationship. Business and society, 36(4), 419-429.

Salazar, J. N. A., \& Benedicto, G. C. (2004). Contabilidade financeira. São Paulo: Pioneira Thompson Learning.

Santos, A. (1999). Demonstração contábil do valor adicionado- DVA: um instrumento para medição da geração e distribuição de riqueza das 
empresas. Tese de Livre Docência, Faculdade de Economia e Contabilidade da Universidade de São Paulo, SP, Brasil.

Santos, A. (2007). Demonstração do valor adicionado: como elaborar e analisar a DVA. São Paulo: Atlas.

Savytzky, T. (2005). Análise de balanços: método prático (3a ed.). Curitiba: Juruá.

Schrickel, W. K. (1999). Demonstrações financeiras: abrindo a caixapreta.São Paulo: Atlas.

Silva, A. A. (2012). Estrutura, análise e interpretação das demonstrações contábeis (3a ed.). São Paulo: Atlas.

Simonsen, M. H. (1994). Ensaios analíticos. Rio de Janeiro: Fundação Getulio Vargas.

Souza, A. A. (2003). A teoria dos jogos e as ciências sociais. Dissertação de Mestrado em Ciencias Sociais, Faculdade de Filosofia e Ciências da Universidade Estadual Paulista Júlio de Mesquita Filho, Marília, São Paulo, Brasil.

Ullmann, A. A. (1985). Data in search of a theory: a critical examination of the relationships among social performance, social disclosure, and economic performance of U.S. firms. Academy of Management Review, $10(3), 540-557$.

Von Neumann, J., \& Morgenstern, O. (1953). Theory of games and economic behavior (3rd ed.). Princeton: Princeton University Press.

World Comission on Environment and Development - WCED. (1987). Our common future. Oxford: Oxford University Press. 